\title{
Designer oils for better nutrition
}

\author{
Anthony J. Kinney
}

The genetic engineering of the fatty acid composition of plants has become a reality. With the right genes and promoters, it is now possible to redesign the fatty acid profile of either plant membranes or plant storage oils. In this issue of Nature Biotechnology, Toguri and colleagues ${ }^{1}$ (pp 1003-1006) describe the expression in tobacco of a cyanobacterial desaturase that introduces a double bond into the $\Delta 9$ position of 16-chain and 18-chain saturated fatty acids. In their transgenic tobacco, there is significant reduction in saturates in both leaf and root, an effect that they correlate with an increased chilling resistance of the plants.

For many, however, the words "reduced saturates" immediately bring to mind the health issues associated with the consumption of edible oils. Expressing the cyanobacterial $\Delta 9$ desaturase in seeds, rather than leaf or root, would be one possible way of reducing the saturate content of vegetable oil, thus improving its nutritional qualities. Indeed, other groups have attempted this very tactic, using either yeast ${ }^{2}$ or $\operatorname{rat}^{3} \Delta 9$ desaturases. A recent report describes the expression of another cyanobacterial desaturase, this time a $\Delta 6$ enzyme, in tobacco ${ }^{4}$. The $\Delta 6$ desaturase introduces a third double bond into linoleic acid, which is abundant in most plant oils, to produce $\gamma$-linolenic acid. This latter compound is found mainly in fish oils and is thought to help alleviate hypocholesterolemia.

Another nutritional concern related to edible oils is the effect of so-called trans fatty acids. These are predominantly the trans-isomers of oleic acid, which are not normally found in plant oils but are produced when the oil is hydrogenated. Most of the vegetable oil produced for commercial food applications is hydrogenated to increase its oxidative stability during storage or frying, or to make the oil solid for edible spreads and margarines. For a number of years now, the consumption of trans fatty acids has been regarded as a possible risk factor for coronary heart disease ${ }^{5}$.

These concerns may be best overcome by the use of desaturase genes from the native plant rather than genes from cyanobacteria. For example, at DuPont (Wilmington, DE), we have cloned an $\Omega 6$ desaturase from soybean, which inserts a second double bond into oleic $\mathrm{acid}^{6}$, and have used this to suppress the endogenous soybean gene. This results in an almost complete elimination of polyunsaturat-

Anthony J. Kinney is senior research biochemist at DuPont Experimental Station, P.O. Box 80402, Wilmington, DE 19880-0402

(kinneya@a1.esvax.umc.dupont.com). ed fatty acids in soybean oil. The first largescale samples of this oil for test marketing will be available by the end of the year?. Similarly, Calgene (Davis, CA) has reported the suppression of an endogenous $\Delta 9$ desaturase to produce a high stearate canola oil for possible use in margarine and other solid fat applications ${ }^{8}$. Both these oils are free from trans fatty acids and should provide significant health benefits once they are widely available.

However, an acyl-ACP (acyl carrier protein) thioesterase gene, rather than a desaturase, has resulted in the first commercially

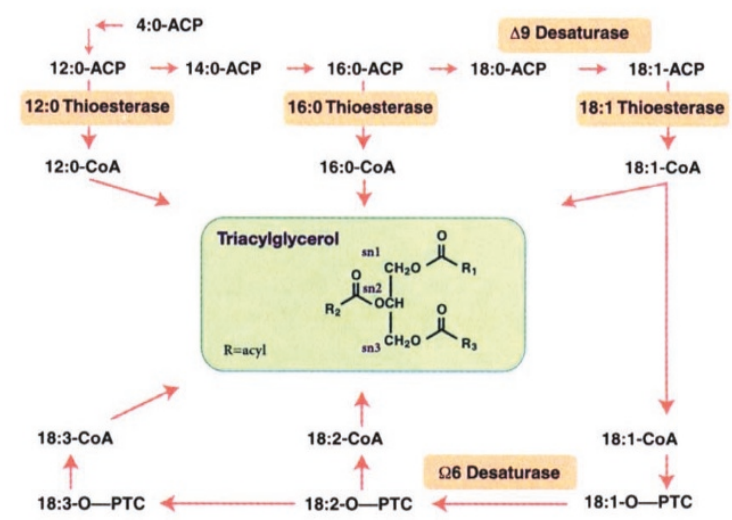

Figure 1. The many paths to triacylglycerol biosynthesis in the developing oilseed. Fatty acyl chains are extended while they are attached to ACP. $\Delta 9$ desaturase may introduce a double bond into 18:0-ACP to form oleate. Thioesterases terminate the elongation process by releasing acyl chains from ACP. The free fatty acids are converted to fatty acyl-CoAs, which are then attached to the three carbons of glycerol by acyltransferases to make triacylglycerol.

marketed "designer oil" for human consumption. The new oil is Calgene's high laurate canola, currently being sold for use in non-dairy creamers and confectionary coatings as a nutritionally improved substitute for palm kernel oil'.

The specificity of acyl-ACP thioesterases determines the ultimate chain length of fatty acids in plant oils. In the developing oilseed, fatty acyl chains are elongated from 4 carbon molecules up to 18 carbons while they are attached to ACP. Acyl-ACP thioesterases release newly synthesized saturated and monounstaurated fatty acid chains from ACP and make them available for further desaturation or incorporation into triacylglycerol.

Most of the common oilseeds, such as soybean and canola, have thioesterases that release only 16 -carbon and 18-carbon chains, and these are the fatty acid chain lengths found in the oil. Researchers at Calgene have isolated a gene from California bay laurel encoding a thioesterase that releases 12 -carbon (lauryl) acyl chains. When they expressed the bay laurel gene in canola, the resulting oil contained up to $50 \%$ lauric acid ${ }^{9}$.

The improved nutritional qualities of the new oil result from the way the transgenic canola incorporates 12-carbon acyl chains into triacylglycerol, putting them only on the first and third carbons (the snl and sn 3 positions) of the glycerol molecule, with an oleate chain on the second carbon (sn2 position). Palm kernel has about $50 \%$ of its total laurate at position sn2. When fats are digested by animals, the outer two fatty acids are first removed, and then the fat is absorbed as a sn2-monoacylglycerol. Unlike sn2-monoacylglycerols containing laurate, those containing oleate have not been linked to heart disease.

This same group has now isolated thioesterases from different plants that have activity toward 8-carbon and 10-carbon acyl-ACPs ${ }^{10}$ and has even begun to custom modify the specificity of thioesterases by protein engineering ${ }^{11}$. The aim is to produce structured triacylglycerols with fatty acid chains at the sn 1 and $s n 3$ position that are even shorter than 12 carbons. These oils are currently made only by expensive transesterification methods and have numerous nutritional applications, including infant formulas, intravenous feeding, and athletic supplements.

Ironically, acyl-ACP thioesterases may turn out to be more useful than desaturases for reducing saturates. For example, our group at DuPont have recently been able to reduce the saturate content of soybean oil by more than two-thirds by blocking the expression of soybean's own 16-carbon specific thioesterase ${ }^{7}$.

\footnotetext{
1. Ishizaki-Nishizawa, O. et al. 1996. Nat. Biotechnol. 14:1003-1006.

2. Polashock, J.J. et al. 1992. Plant Physiol. 100:894-901.

3. Torisky, R., et al. 1995. Plant Physiol. 108(s):128.

4. Reddy, A.S. et al. 1996. Nat. Biotechnol. 14:639-642.

5. Allen, A.H. 1995. Food Prod. Des. 5:30-49.

6. Heppard, E.P. et al. 1996. Plant Physiol. 110:311-319.

7. Kinney, A.J. Oral Presention at the 12th International Symposium on Plant Lipids, Toronto, July 7-12, 1996.

8. Knutzon, D.S. et al. 1992. Proc. Natl. Acad. Sci. USA 89:2624-2628

8. Del Vecchio, A.J. 1996. Inform. 7:230-243.

10. Dehesh, K. et al. 1996. Plant J. 9:167-172.

11. Yuan, Let al. 1995. Proc. Natl. Acad. Sci. USA
} 92:10639-10643. 